\title{
17-month-old child with Pearson syndrome and corneal haze - case report
}

\author{
Katarzyna Dziedziech, Dominik Śliwiak, Małgorzata Woś \\ The Ophthalmology Ward with Pediatric Ophthalmology Unit of the "Stefan Żeromski" Tertiary Hospital in Krakow, Poland
}

\section{ABSTRACT}

The article presents a case of a 17-month-old boy with bilateral corneal haze in the course of Pearson syndrome - a rare mitochondrial disease. In this paper the cause, course and ophthalmologic changes in this disease have been cited and discussed.

KEY WORDS: Pearson disease, Pearson syndrome, corneal haze, rare disease, mitochondrial disease.

\section{INTRODUCTION}

The aim of the article is to present the clinical case of a 17-month-old child with Pearson's syndrome and concomitant changes in the organ of vision.

Pearson's syndrome is a rare, systemic, fatal mitochondrial disease (common deletion of $4977 \mathrm{bp}$ mtDNA designated m.8470_13446del4977) [1], damaging many systemic organs, including the cornea, liver, pancreas, kidneys, hematopoietic system and the central nervous system.

To date, about 100-150 cases of the disease have been described worldwide - for the first time in 1979 by Howard Pearson, and the incidence in Italy has been described as 1 case per million births [2]. The disease is inherited from the mother by mitochondrial DNA. The most common symptom observed at the beginning of the disease is sideroblastic anemia (an increase in the number of abnormal sideroblasts due to a disorder of heme activity), requiring multiple blood transfusions during the child's life [3].

The most commonly reported ophthalmic symptom is corneal opacity, most likely secondary to endothelial cell failure. Other symptoms include congenital cataract with secondary development of amblyopia and strabismus, retinopathy of prematurity, and cases of congenital drooping eyelids (as a forecast of the development of Kearns-Sayre syndrome). Other systemic symptoms associated with the syndrome include: neutropenia, thrombocytopenia, leukopenia, lactic acidosis, tubulopathy, encephalopathy, neonatal diabetes, exocrine pancreatic insufficiency, and growth retardation [4-7].

Transformation of Pearson syndrome into Leigh syndrome (subacute necrotic encephalopathy) or Kearns-Sayre syndrome (Pearson and Kearns-Sayre syndrome are caused by the same mutation in mitochondrial DNA $[8,9])$ has been observed in some cases.

To make the diagnosis of the disease, bone marrow biopsy should be performed, showing vacuolization of precursor cells and presence of ring sideroblasts. It has been shown that biopsy done after the first month of life gives more information about the changes taking place in the bone marrow than the same procedure carried out just after birth. However, due to the high variability in test results and non-characteristic symptoms in the neonatal period, that may suggest presence of a different disease, the only way to confirm the syndrome is by mitochondrial DNA analysis. For this purpose, leukocyte DNA is subjected to the Southern-Blot analysis [10]. The prognosis is very unfavorable, most children with this syndrome die in infancy or early childhood due to severe infections as well as metabolic disorders and multiple organ failure. Although the disease requires cooperation and involvement of a number of specialists in the diagnostic and therapeutic process, most children do not reach the age of 3 years.

Due to a more detailed understanding of the etiology of mitochondrial diseases and the association of Pearson's syndrome with Kearns-Sayre and Leigh syndromes, and above all, the possibility of their treatment, comfort of life of these patients has significantly improved, and resulted in an extension of the average life expectancy, even up to 8 years [11].

\section{CASE REPORT}

A mother with a 17-month-old baby boy reported to the Ophthalmology Outpatient Clinic of the "Stefan Żeromski" Hospital in Krakow, for consultation. The patient had been referred from the GP's office because of bilateral hazing of cor- 


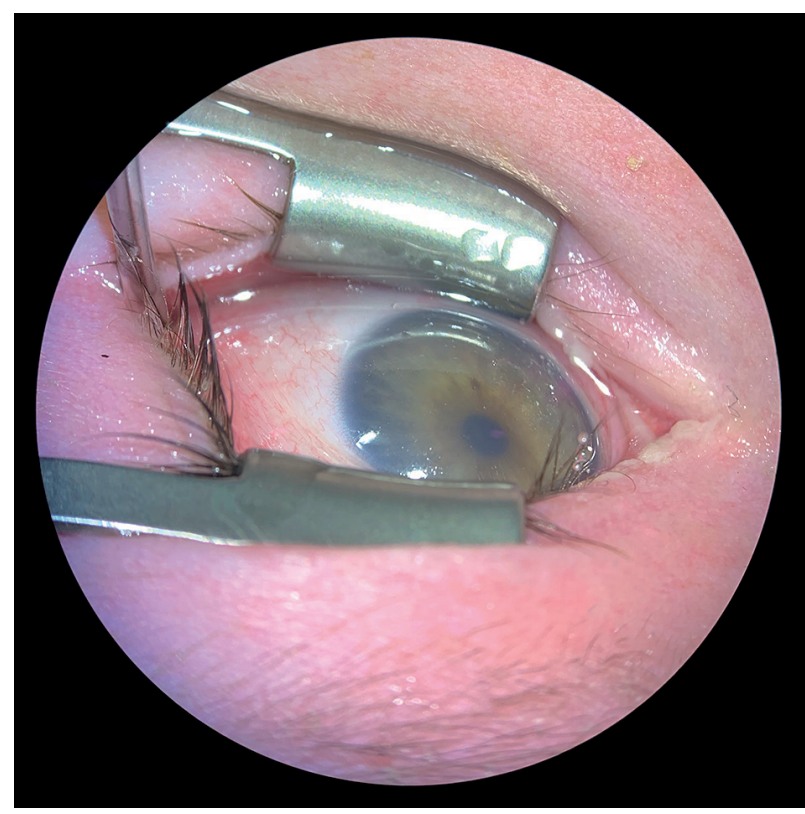

Figure 1. Corneal opacity of the left eye - three months after the treatment

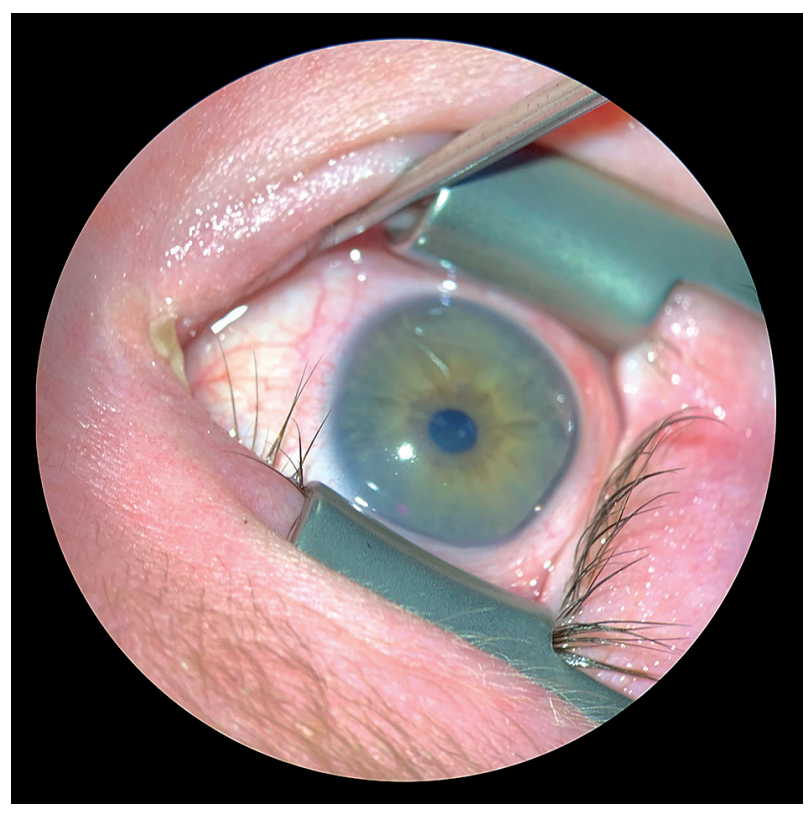

Figure 2. Poor corneal translucency of the right eye - three months after the treatment

Fison's speculum was used. An attempt was also made to study the morphology and number of endothelial cells using a mirror-illuminated microscope. Because the child did not cooperate, the study could not be carried out objectively. Because of persistent inflammation of the conjunctiva in the right eye and hazing of the corneal parenchyma in both eyes, a decision was made to maintain administration of ofloxacin 4 times daily, 1\% tropicamide 3 times daily (for 4 weeks), topical dexamethasone 2 times daily (in the form of drops with zero content of preservatives) and moisturizing drops with trehalose to both eyes.

After about 4 weeks, a gradual regression of inflammation with persistent nummular corneal haze in the right eye were observed. Ofloxacin drops were discontinued, dexamethasone was maintained to be administered once daily (for 3 months). The child was under constant supervision of the Ophthalmological Outpatient Clinic for about half a year - during that time the inflammation completely receded, nummular inflammation in the right eye disappeared (which most likely suggested bacterial etiology) and no reduction in corneal haze in both eyes was observed. No other abnormalities on the part of the eye were observed during follow-up visits. Parents failed to report with the child for subsequent follow-up visits.

\section{DISCUSSION}

It is difficult to explain whether in the presented case haze and keratitis could had resulted from endothelial cell failure or pathology. Because of a very rare occurrence (about 150 cases in the world, 2 cases in Poland), the available medical literature on Pearson's syndrome only describes single cases of corneal haze and corneal endothelial failure, but in children at later age, which was associated with transformation into the Kearns-Sayre syndrome. The described corneal opacity could most likely result from metabolic failure of the endothelial 
pump [12]. In addition, we do not know how can corneal haze in Pearson's syndrome affect the shaping of the vision process and the possible development of amblyopia.

\section{CONCLUSIONS}

Because of its rarity, Pearson's syndrome is often overlooked in the diagnosis of newborns and infants, or confused with other diseases, so in case of refractory anemia observed in a newborn, a physician should always consider the mitochondrial inherited disease.

To sum up - most of ophthalmologic changes in the course of Pearson's syndrome are associated with conversion into Kearns-Sayre syndrome at various age, and associated with the oc- currence of e.g. myopathy, optic neuropathy, retinitis pigmentosa and ophthalmoplegia [13]. Therefore, despite the very rare incidence, all patients with Pearson's syndrome should undergo detailed assessment and ongoing ophthalmologic monitoring. There is currently no causal treatment for the disease, but associated conditions are treated. There are insufficient data in the available literature to explain the exact pathomechanism and indicate long-term effects of corneal haze. No algorithm for treatment and ophthalmological management of these children has been developed.

\section{DISCLOSURE}

The authors declare no conflict of interest.

\section{References}

1. Margaret PA, D'Mura A, Ardinger H, et al. Mitochondrial DNA delation syndromes. GeneReviews. University of Washington, Seattle, June 3, 2013.

2. Manea EM, Leverger $G$, Bellmann F, et al. Pearson syndrome in the neonatal period: Two case reports and review of the literature. J Pediatr Hematol Oncol 2009; 31: 947-951.

3. Pearson HA, Lobel JS, Kocoshis SA, et al. A new syndrome of refractory sideroblastic anemia with vacuolization of marrow precursors and exocrine pancreatic dysfunction. J Pediatr 1979; 95: 976-984.

4. Morel AS, Joris N, Meuli R, et al. Early neurological impairment and severe anemia in a newborn with Pearson syndrome. Eur J Pediatr 2009; $168: 311-315$.

5. Williams TB, Daniels M, Puthenveetil G, et al. Pearson syndrome: Unique endocrine manifestations including Neonatal Diabetes and adrenal insufficiency. Mol Genet Metab 2012; 106: 104-107.

6. Cursiefen C, Küchle M, Scheurlen W, Naumann G0. Bilateral zonular cataract associated with the mitochondrial cytopathy of pearson syndrome. Am J Ophthalmol 1998; 125: 260-261.

7. Farruggia P, Di Cataldo A, Pinto RM, et al. Pearson syndrome: a retrospective cohort study from the Marrow Failure Study Group of A.I.E.O.P. (Associazione Italiana Emato-Oncologia Pediatrica). JIMD Rep 2016; 26: 37-43.

8. Lee HF, Lee HJ, Chi CS, et al. The neurological evolution of Pearson syndrome: Case report and literature review. Eur J Paediatr Neurol 2007; 11: 208-214.

9. Becher MW, Wills ML, Noll WW, et al. Kearns-Sayre syndrome with features of Pearson's marrow-pancreas syndrome and a novel 2905-base pair mitochondrial DNA deletion. Hum Pathol 1999; 30: 577-581.

10. Tadiotto E, Maines E, Degani D, et al. Bone marrow features in Pearson syndrome with neonatal onset: A case report and review of the literature. Pediatr Blood Cancer 2018; 65: 10-13.

11. Broomfield A, Sweeney MG, Woodware CE, et al. Paediatric single mitochondrial DNA deletion disorders: an overlapping spectrum of disease. J Inherit Metab Dis 2015; 38: 445-457.

12. Kasbekar SA, Gonzalez-Martin JA, Shafiq AE, et al. Corneal endothelial dysfunction in Pearson syndrome. Ophthalmic Genet 2013; 34: 55-57.

13. Goldstein A, Falk MJ (eds.). In: Adam MP, Ardinger HH, Pagon RA, et al. (eds.). Mitochondrial DNA deletion syndromes. GeneReviews ${ }^{\circledast}$. University of Washington, Seattle 1993-2019 [access: 31.01.2019]. 